\title{
Benefits and Drawbacks of Regenerative Medicine in Japan
}

\author{
Miyako Takagi
}

\begin{abstract}
Aiming at further development of the regenerative medicine in Japan, ethical investigation in this field was performed. Embryonic stem (ES) cells and induced pluripotent stem (iPS) cells can be induced to differentiate into a wide range of tissues that could be used for therapeutic applications in regenerative medicine. To obtain ES cells, the early embryo has to be destroyed. In contrast, the creation of iPS cells which were first generated in $\mathbf{2 0 0 6}$ does not involve the use or destruction of human embryo. In September 2014, a team in RIKEN Center for Developmental Biology (CDB) transplanted retinal cells produced from iPS cells into the eyes of a woman suffering from macular degeneration. Other trials of iPS cell-based treatments plan to start testing for Parkinson's disease and serious heart failure. On the other hand, the case of stimulus-triggered acquisition of pluripotency (STAP) cells, which is said to be the world's three big cases of scientific misconduct over the last $\mathbf{5 0}$ years, happened in RIKEN/CDB. A female scientist announced that she discovered a new method for creating pluripotent stem cells in mice in January 2014, however it was faked data. As a result, RIKEN/CDB ceased to be a research center in August 2014, and the reputation of Japan's scientific research community was damaged. An important lesson from this case is the necessity of appropriate ethical training on good scientific practices.
\end{abstract}

Index Terms-Clinical trial, iPS cells, regenerative medicine, scientific misconduct.

\section{INTRODUCTION}

Regenerative medicine is the process of regenerating human cells, tissues, or organs to restore or establish normal function. If a regenerated organ's cells were derived from the patient's own tissues or cells, this would potentially solve the problem of the shortage of organs available for donation and the problem of organ transplant rejection. ES cells and iPS cells can be induced to differentiate into a wide range of tissues that could be used for therapeutic applications in regenerative medicine. In September 2014, a team led by RIKEN ophthalmologist M. Takahashi transplanted retinal cells produced from iPS cells into the eyes of a patient suffering from macular degeneration [1]. This patient received the world's first transplant of a retinal sheet made using iPS cells. The government has listed regenerative medicine as a leading light of its growth strategy. It is expected that the demand for medical products in Japanese regenerative medicine will grow to approximately a trillion yen in 2030.

On the other hand, also in 2014, one of the world's three

Manuscript received March 4, 2015; revised June 5, 2015.

Miyako Takagi is with the University Research Center, Nihon University, 4-8-24, Kudan-minami, Chiyoda-ku, Tokyo, 102-8275, Japan (e-mail: takagi.miyako@ nihon-u.ac.jp). big cases of research misconducts over the last 50 years occurred in the field of regenerative medicine. Why did this happen? One reason was that the publish-or-perish pressure in the scientific race led to the acceptance of false data by a prestigious journal. In order to avoid research misconduct, ethical education is now necessary more than ever.

\section{METHODS}

We collected published documents relating to regenerative medicine, and analyzed them for the ethical issue.

\section{ES AND IPS CELLS}

ES cells are pluripotent, that is, they are able to differentiate into all derivatives of the three primary germ layers: ectoderm, endoderm, and mesoderm. These include each of the more than 220 cell types in the adult body. Additionally, under defined conditions, ES cells are capable of indefinitely propagating themselves. This allows ES cells to be employed as useful tools for regenerative medicine. However, to obtain ES cells, the early embryo has to be destroyed. This results in destroying a potential human life, which raises serious ethical concerns.

iPS cells, which were first generated in 2006 by Kyoto University's Professor Yamanaka - who won the Nobel Prize in 2012 for these achievements — are a type of pluripotent stem cell artificially derived from an adult somatic cell that are created by inducing forced expression of certain genes [2]. iPS cells are believed to be identical to natural ES cells in many respects, such as the expression of certain stem cell genes and proteins and their ability to differentiate. They are also less prone to immune rejection than ES cells because they are derived entirely from the patient. iPS cells do not involve the use or destruction of human embryos; therefore, researchers believe that iPS cell research leads the field of new regenerative medicine without the controversial use of embryos. For safety, reprograming of adult cells to obtain iPS cells may pose some risk, but it is improving day by day.

\section{BenEFITS - REgENERATIVE MEdicine Moves to REALITY}

\section{A. Epoch-Making Treatment Using iPS Cells}

Approximately 700,000 Japanese individuals are estimated to be suffering from age-related macular degeneration, the symptoms of which include distorted vision. The current treatment is symptomatic therapy to prevent the patient's condition from further deteriorating. In September 2014, a 
team led by RIKEN ophthalmologist, Dr. M. Takahashi transplanted retinal cells produced from iPS cells into the eyes of a woman suffering from this disease. This patient received the world's first transplant of a retinal sheet made using iPS cells. This clinical trial is the first step of the epoch-making research that could further evolve regenerative medicine. With the transplant of retinal cells, the patient could have low vision. In other words, the trial may be able to halt the patient's visual degradation, but it should not be expected to cause any dramatic recovery of vision. Even so, expectations among patients to the effectiveness of such a transplant are believed to be high.

This surgical procedure carries important implications as a clinical study to confirm the safety of medical technology using iPS cells. The patient is said to be doing well. The number of patients in the clinical trial will be six. Scientists still have much to learn about how iPS cells behave once implanted in the body.

Over the past eight years since Professor S. Yamanaka produced human iPS cells, the risk of iPS cells becoming cancerous has almost been resolved, while the efficiency of their production has been enhanced. The risk that iPS cells cancerate and any other unknown side effects will be examined over the next four years. Cost is another hurdle to be overcome. It took approximately 10 months and tens of millions yen to create the sheets of iPS cells that went into the patient's eyes. In order to develop it for general treatment, the Kobe city government and affiliated organizations plan to build a new research base for regenerative retina treatments using iPS cells.

\section{B. Other Trials of iPS Cell-Based Treatments}

Other trials of iPS cell-based treatments are in progress. In the next two years, a team at Kyoto University will try out a remedy for Parkinson's disease [3], whereas Osaka University scientists will conduct an experiment aimed at relieving heart failure [4].

Researchers at Kyoto University have devised a technique to create neurons from iPS cells, paving the way for clinical testing to treat Parkinson's disease by 2016. This team used blood from patients with degenerative brain disease to create iPS cells, differentiated them into a large number of neurons, and inserted them into participants' brains. The goal is to raise levels of the neurotransmitter dopamine. The team has verified the therapeutic effects of implanted neurons derived from iPS cells in tests on mice and primates. They aim to submit a proposal for the human trial, which will include six participants. Once the human trial is underway, researchers expect it to take approximately nine months to confirm that the artificially created neurons are safe for implantation.

There are an estimated 100,000 Japanese individuals with serious heart failure. The group at Osaka University will start clinical trials on restoring the heart functions of patients with serious heart failure by placing a medicine patch created from iPS cells on the heart. The medicine patch is expected to prompt the rejuvenation of blood vessels and cardiac muscle cells. The clinical trials will start in June 2015, and the hospital will aim to obtain approval for the treatment by 2021 .

The therapy uses an antithrombotic agent (TS1402) developed more than 20 years ago. The agent did not appear on the market because of side effects, but a team at Osaka University discovered that it is effective in treating heart failure. The therapy reached the clinical trial stage by processing the agent into a medicine patch, which weakened the side effects. Twenty-four patients with ischemic cardiomyopathy, a disease that causes some heart muscles to malfunction because of myocardial infarction and other factors, will participate in the clinical trial. The research group will assess the safety and efficacy of the treatment.

Besides these clinical trials, Keio University plans to start testing a fix for spinal cord damage in 2017-18. Treatments for diabetes, leukemia, and muscular dystrophy are moving toward the trial stage.

\section{The New Regulation for the Safety in Medical Practical Application}

As regenerative medicine is still in its early stages of development, it is important to verify the safety of such practical applications. The law for securing the safety of regenerative medicine has been newly established to ensure the quality of medical treatment. It took effect in November 2014 [5].

When we hear "regenerative medicine," we think of medical treatment using ES and iPS cells. However, there is a wide range of regenerative medicine, including a method of using somatic stem cells extracted from the patient's own body. The injection of immune cells to treat cancer also falls under the category of cell transplantation.

Under the new law in regenerative medicine, all types of cell remedies are subject to regulations. It is appropriate to apply different regulations to different types of cells, considering the risks involving the cells and data that have been accumulated widely differ depending on each type of cell. The use of processed cells, such as iPS and ES cells, which have never been clinically applied, would require permission from the health, labor, and welfare minister. Medical institutions that intend to transplant somatic stem cells and use immunotherapy, safety of which has been partially confirmed, must notify the authorities after the methods are screened by a third-party panel.

Many cosmetic surgery clinics in Japan are believed to be conducting stem-cell injections for anti-aging treatment. If these treatments are left uncontrolled, they could eventually lead to serious accidents, adversely affecting the promotion of regenerative medicine. The ministry drew up an outline of a bill aimed at ensuring the safety of regenerative medicine involving cell injections.

The law requires medical institutions providing regenerative medicine to submit treatment plans to government-authorized committees for screening. Violators will be punished. Such committees, which are to be established by university hospitals and academic societies, will comprise doctors, lawyers, bioethicists, and others. With an expanded net of regulation, medical treatments whose safety has not been established can be eliminated in the evaluation process.

For tissue-engineered medical products, a new system has been introduced to allow their conditional approval for a designated period of time once their safety has been confirmed after a certain minimum number of clinical trials 
and their effectiveness has been assured.

The government has listed regenerative medicine as a pillar of its growth strategy. It is expected that the demand for medical products in regenerative medicine, such as organ cells grown from iPS cells, will be approximately one trillion yen in 2030 [6], including medical treatments using the products and related businesses such as devices and containers to culture iPS cells. To expand the market in this field, it is critical to win the public's confidence.

\section{DRAWBACKS - ONE OF THE THREE BIG CASES OF SCIENTIFIC MISCONDUCT OVER THE LAST 50 YEARS}

It is said that the case of STAP cells, the case of Hwang Woo-Suk, and the Schön affair are the world's three big cases of scientific misconduct over the last 50 years.

\section{A. Case of Ms. Obokata in RIKEN/CDB, Japan (2014)}

Ms. Obokata from the RIKEN/CDB was hailed as a "heroine" of female scientists because of her discovery of a new method for creating pluripotent stem cells in mice, known as STAP, in January 2014 [7]. The studies, which were published in the January 30, 2014 edition of the scientific journal Nature, were conducted by a team led by Ms. Obokata. Scientists over the world were astonished at the finding that pluripotency is believed to be unique to germ cells. ES cells are harvested from fertilized eggs and destroy the embryo in the process. In the creation of iPS cells, four types of genes are introduced into somatic cells using viral vectors to forcibly reset them, reverting the cells to a state resembling that of a fertilized egg. In making STAP cells, however, somatic cells can be reset by just placing them in a weak acid bath. Unlike ES cells, STAP cells are produced without destroying fertilized eggs, a subject of ethical concern. In addition, STAP cells are unlikely to turn cancerous in the body, which is a problem with iPS cells. RIKEN has applied for an international patent for the process of creating STAP cells.

However, two weeks after the amazing announcement of STAP cells, suspicious points of this study were indicated on the internet. Immediately, RIKEN created an investigation committee. In April, the investigation committee announced that Ms. Obokata committed two counts of scientific misconduct, leading to the retraction of the studies by Nature in July. In August, Dr. Y. Sasai, the CDB deputy director committed suicide. He had served as an adviser to Ms. Obokata and as co-author of the papers. In December 2014, the investigation committee concluded that STAP cells were derived from ES cells based on examinations of the remaining samples [8], but the panel was unable to determine who introduced ES cells, and whether it happened deliberately or by accident citing insufficient evidence. As a result of Ms. Obokata's misconduct, RIKEN/CDB ceased to be a research center in August 2014, and the reputation of Japan's scientific research community was damaged.

B. Case of Hwang Woo-Suk of Seoul National University, South Korea (2005)

In 2004, a Korean scientist, Hwang Woo-Suk, announced his success in the world's first human cloning experiments.
Dolly the Sheep, famous for being the first successfully cloned mammal in 1997, was created using the process of somatic cell nuclear transfer (SCNT) [9]. SCNT is a technique for creating an embryo from a somatic cell and an egg cell. The technique consists of taking an enucleated oocyte and implanting a donor nucleus from a somatic cell.

Considered a national hero, he had surprised the world by reporting the creation of 11 patient-specific stem cell lines in 2005 by SCNT [10]. However, in 2006, his research activities were halted when it emerged that many of his data on SCNT were fabricated. His team also committed several ethical violations during their research. He worked with an enormous number of eggs — 2,061 from 129 women — despite claiming to have used only 242 eggs for the 2004 study and 185 for the 2005 study. Investigations revealed that many of the women who provided eggs had not given valid informed consent and that nearly $75 \%$ of them reported that they were given cash or enticed by various financial incentives. In 2009, Hwang was convicted of embezzling research funds and illegally buying human eggs for his research [11]. The findings were a huge setback for human cloning using SCNT. Even after using a large number of human eggs, he was unable to derive such stem cells, and the field is now left with no evidence that it is possible in humans.

\section{Case of Jan Hendrik Schön at Bell Labs, USA (2002)}

Jan Hendrik Schön, a German physicist, began working at Bell Labs, New Jersey, USA, in 1997. He had worked in nanotechnology and briefly rose to prominence after a series of apparent breakthroughs with semiconductors [12]. He published numerous papers in rapid succession in high-profile journals, including Nature and Science. He was considered a star scientist, and his colleagues were beginning to tip him for a Nobel Prize.

Scientists, however, were unable to reproduce Schön's results. In 2002, it was found that graphs in three unrelated papers appeared identical down to what should have been random noise. Bell Labs began an investigation on the subject of the validity of Schön's work. They found that Schön removed data points that disagreed with predictions and even used mathematical functions in place of real data points, and at least 16 papers falsified data while he was a researcher at Bell Labs. According to the report, most of the evidence of those original experiments vanished. Schön was dismissed from Bell Labs, and two years later, the University of Constance in Germany decided to revoke the $\mathrm{PhD}$ he had been awarded in 1998 [13].

\section{THE SimILARITY OF THE BACKGROUND IN THE SCHÖN AFFAIR AND THE STAP CELLS' CASE}

Around 2000, the parent company of Bell Labs had financial difficulties; research funds were reduced and the number of researchers decreased. Therefore, a star researcher was strongly desired by the executives of Bell Labs. That was one reason why the internal checks on Schön were loose. The parent company ultimately terminated a basic physical study section in Bell Labs six years after the Schön affair. This background is similar to the STAP cells' case [14].

The Japanese government had decided to support 
regenerative medical research with 110 billion yen from 2013 to 2023. RIKEN had a sense of impending crisis that most of the government budgets would be invested in the iPS cells research of Kyoto University. In order to attract government funds, the demand for RIKEN to produce non-iPS cell research grew, leading to a loosening of its internal checks. The government finally decided that RIKEN/CDB would cease to be a research center in August 2014. The excessive expectations for new findings may lead to scientific misconduct.

\section{THE NECESSITY OF ETHICAL EDUCATION IN ORDER TO AVOID SCIENTIFIC MISCONDUCT}

A 2012 study [15] found that the long-term trend for misconduct was on the upswing: in 1976, there were only three retractions for misconduct out of 309,800 papers $(0.00097 \%)$, whereas there were 83 retractions for misconduct out of 867,700 papers at a recent peak in 2007 $(0.0096 \%)$. Over these 30 years, there was a tenfold percentage increase in scientific research papers being retracted for fraud. There are a few reasons why retractions and fraud have increased. One reason is that the publish-or-perish pressure in the scientific race may induce a researcher to produce false data in order to be accepted in a prestigious journal. Another view suggests that because journals are now published online and are more accessible to a wider audience, it is easier for experts to find fraudulent papers. In Schön's case, it was two years before he was accused of scientific misconduct. On the other hand, in the case of STAP cells, the scientifically suspicious data in this research were pointed out on the internet only one week after the study was published online in 2014.

Another important point for research is the laboratory notebook [16]. A laboratory notebook is a primary record research. Researchers use a laboratory notebook to document their hypotheses, experiments, and initial analysis or interpretation of these experiments. It must be written regularly by the researcher as an honest representation of the research work conducted. The main researcher in STAP cells, Ms. Obokata, had very few descriptions in her notebook, and no one understood how her study was advancing except her. Therefore, the investigation committee was not able to understand how she performed the experiments. A research leader may be requested to check the researchers' laboratory notebook.

An important lesson from these cases is the necessity of appropriate ethical training on good scientific practices. In Japanese scientific institutes and universities, more ethical training should be necessary in graduate through postdoctoral studies.

\section{ACTIONS OF THE USA FOR SCIENTIFIC MISCONDUCT}

The Office of Research Integrity (ORI) [17] handles allegations of scientific misconduct in the USA. Scientific misconduct includes fabrication, falsification, or plagiarism. It does not include honest error or honest differences in the interpretation or judgment of data.
In an investigation process, a person reports possible misconduct in scientific research to a Scientific Director (SD). The SD will consult with the National Institutes of Health (NIH) Research Integrity Officer and assess the allegation. If this assessment suggests sufficient evidence or information to support the need for further evaluation, ORI establishes a committee to carry out a formal inquiry. At this point, the Inquiry Committee secures all notebooks, records, and data related to the case for review. During the inquiry, the Committee gathers information to determine if the alleged conduct is within the definition of misconduct in science and substantial enough to allow a specific finding of scientific misconduct. ORI recommends the findings and sanctions the Assistant Secretary for Health who makes the final decision regarding the scientific misconduct. Final decisions are subject to appeal through the Departmental Appeals Board (DAB). There is no such national office in Japan. It is necessary to set up a place where people can report possible scientific misconduct.

\section{CONCLUSION}

We hope that the potential of regenerative medicine will be realized in the near future. The first step of this clinical study was already conducted in the transplantation of retinal cells produced from iPS cells to a patient. At some university hospitals and other medical institutions, clinical iPS research approved by the government is steadily progressing. The government has listed regenerative medicine as a leading light of its growth strategy. It is expected that the demand for medical products in regenerative medicine, such as organ cells grown from iPS cells, will be approximately one trillion yen. To expand the market in this field, it is critical to win the public's confidence.

The competition for research funding between researchers and also between research institutes increases very year. The excessive expectation for new findings may lead to scientific misconduct. Important lessons from misconduct cases are a necessity for appropriate ethical training on good scientific practices.

\section{REFERENCES}

[1] M. Takahashi, J. Assawachananont, M. Mandaiemail, S. Okamoto, C Yamada et al., "Transplantation of embryonic and induced pluripotent stem cell-derived 3d retinal sheets into retinal degenerative mice," Stem Cell Reports, vol. 2, no. 5, pp. 662-674, May 2014.

[2] K. Takahashi and S. Yamanaka, "Induction of pluripotent stem cells from mouse embryonic and adult fibroblast cultures by defined factors," Cell, vol. 126, no. 4, p. 663, Sept. 2006.

[3] K. Nishimura and J. Takahashi, "Therapeutic application of stem cell technology toward the treatment of Parkinson's disease," Biol Pharm Bull, vol. 36, no. 2, pp. 171-175, 2013

[4] Y. Sawa, M. Kawamura, S. Miyagawa, K. Miki, A. Saito et al. "Feasibility, safety, and therapeutic efficacy of human induced pluripotent stem cell-derived cardiomyocyte sheets in a porcine ischemic cardiomyopathy model," Circulation, 2015.

[5] Ministry of Health, labor and Welfare. (November 2014). Law about the safety of the regenerative medicine. [Online]. Available: http://www.mhlw.go.jp

[6] Council for Science, Technology and Innovation. (2013) Science-and-technology strategy. [Online]. Available: http://www8.cao.go.jp/cstp/ 
[7] H. Obokata, T. Wakayama, Y. Sasai, K. Kojima, M. P. Vacanti et al., "Stimulus-triggered fate conversion of somatic cells into pluripotency," Nature, vol. 505, pp. 641-647, Jul. 2014.

[8] RIKEN investigation committee. (December 2014). The final search report for STAP cells. [Online]. Available: http://www.riken.jp/pr/topics/2014/20141226_1/

[9] I. Wilmut, A. E. Schnieke, J. McWhir, A. J. Kind, and K. H. S Campbell, "Viable offspring derived from fetal and adult mammalian cells," Nature, vol. 385, no. 6619, pp. 810-613, Jul. 1997.

[10] W. S. Hwang, S. I. Roh, B. C. Lee, S. K. Kang, D. K. Kwon et al., "Patient-specific embryonic stem cells derived from human SCNT blastocysts," Science, vol. 308, no. 5729, pp. 1777-1783, June 2005.

[11] CSMonitor.com, Light Sentence for Disgraced Korean Cloning scientist, The Christian Science Monitor, Oct. 2009.

[12] J. H. Schön, "New phenomena in high mobility organic semiconductors," Basic Solid State Physics, vol. 226, no. 2, pp. 257-270, 2001

[13] Report of the Investigation Committee on the Possibility of Scientific Misconduct in the Work of Hendrik Schön and Coauthors, Sept. 2002

[14] M. Suda, Scientist of the Forgery, pp. 1-383, Dec. 2014
[15] C. Ferric, R. Fang, G. Steen, and A. Casadevall, "Misconduct accounts for the majority of retracted scientific publications," Proc. Natl Acad Sci USA, vol. 109, no. 42, pp. 17028-17033, Oct. 2012.

[16] M. Kühne and A. W. Liehr, "Improving the traditional information management in natural sciences," Data Science Journal, vol. 8, pp $18-26,2009$.

[17] The Office of Research Integrity. [Online]. Available: http://ori.hhs.gov/

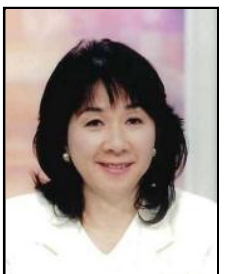

Miyako Takagi is a professor of University Research Center at Nihon University. Her research interests include ethical, legal and psychosocial aspects of regenerative medicine and neuro-science. She is a member of Bioethics Committee in Japanese Cabinet Office, and also she is the president in Asian Bioethics Association. She received her Docteur d'Université ès Sciences, Université Paris VII 